Article

\title{
Analysis of Wind Generator Operations under Unbalanced Voltage Dips in the Light of the Spanish Grid Code
}

\author{
Vicente León-Martínez and Joaquín Montañana-Romeu * \\ Electrical Engineering Department, Universidad Politécnica de Valencia / Camino de Vera, \\ 14. 46022-Valencia, Spain; E-Mail: vleon@die.upv.es \\ * Author to whom correspondence should be addressed; E-Mail: jmontanana@die.upv.es; \\ Tel.: +34-963-879-594; Fax: +34-963-877-599.
}

Received: 29 June 2011; in revised form: 20 July 2011 / Accepted: 22 July 2011 /

Published: 8 August 2011

\begin{abstract}
Operation of doubly fed induction generators subjected to transient unbalanced voltage dips is analyzed in this article to verify the fulfillment of the Spanish grid code. Akagi's p-q theory is not used for this study, because control of the electronic converter is not the main goal of the paper, but rather to know the physical phenomena involved in the wind turbine when voltage dips occur. Hence, the magnetizing reactive power of the induction generators and their components, which are related with the magnetic fields and determine operation of these machines, are expressed through the reactive power formulations established in the technical literature by three well-known approaches: the delayed voltage (DV) method, Czarnecki's Current's Physical Components (CPC) theory and Emanuel's approach. Non-fundamental and negative-sequence components of the magnetizing reactive power are respectively established to define the effects of the distortion and voltage imbalances on the magnetic fields and electromagnetic torques. Also, fundamental-frequency positive-sequence and negative-sequence reactive powers are decomposed into two components: due to the reactive loads and caused by the imbalances. This decomposition provides additional information about the effects of the imbalances on the main magnetic field and electromagnetic torque of the induction generator. All the above mentioned reactive powers are finally applied to one actual wind turbine subjected to a two-phase voltage dip in order to explain its operation under such transient conditions.
\end{abstract}


Keywords: reactive power; unbalanced three-phase systems; grid codes; wind energy; wind power generation

\section{Introduction}

Today, from an environmental viewpoint, it has become extremely important to use wind power for electrical energy generation. Wind farms, which are areas with many wind turbines used for generating electric power, have been established in many countries. It is important to ensure continuous operations of these wind farms so that the power supply can be constant and reliable. Hence, grid codes have been established in many countries to avoid unexpected wind-farm disconnections, which can perturb the stability and operations of the electrical network. Sudden wind-farm disconnections occur because of dips in transitory parameters such as voltages. In Section 2 of this article, it will be seen that according to the Spanish grid code requirements [1,2], wind-generators must not only not have any disconnections, but they must also deliver reactive powers to the electrical network during any disturbances. The Spanish grid code also indicates the maximum allowed reactive power consumptions in the perturbation and the standard IEC 61400-21 [3] specifies that the mentioned reactive power must be the fundamental-frequency positive-sequence reactive power (FPRP), established by several theories [4-6] and included in the IEEE Standard 1459 [7].

The main magnetic field of the wind generators under any voltage conditions is defined on the basis of FPRP $\left(Q_{+}\right)$. This magnetic field governs the voltages and electromechanical stability of these machines in the normal operation, but when voltages are unbalanced and/or distorted, as occurs during voltage dips, the magnetic field is deformed and operation of the wind generators cannot be explained only by means of FPRP. New reactive power components corresponding to each involved phenomena must be considered jointly with the FPRP to accurately analyze operation of wind turbines subjected to voltage dips. Several reactive power formulations are known in the technical literature and could be used for this purpose. Delayed voltage (DV) reactive power and CPC fundamental reactive power, described by Czarnecki in [8], are used in Section 3 to obtain other reactive power components which can help us to analyze the magnetic field of the wind generators in the case of unbalanced and distorted voltages. Non-fundamental reactive power $\left(Q_{H}\right)$ is established by comparing DV reactive power and CPC fundamental reactive power. This reactive power component defines the distorted magnetic field superposed on the main magnetic field of the wind generators as a result of the voltage distortion. Fundamental negative reactive power $\left(Q_{-}\right)$is obtained as the difference between CPC fundamental reactive power and FPRP. Last reactive power characterizes the reverse magnetic fields superposed to the main magnetic field, which are caused by the voltage unbalances and disturb wind generators operations. Negative reactive power $\left(Q_{-}\right)$is decomposed into the reactive powers due to the reactive loads $\left(Q_{r^{-}}\right)$and caused by the imbalances $\left(Q_{u^{-}}\right)$. Also, in Section 3, FPRP is divided into two reactive power components [9-11]: due to the reactive loads (with balanced voltages), $Q_{r+}$, and caused by the imbalances, $Q_{u^{+}}$. These decompositions can be very useful to analyze wind generator operations and to explain some of their poor workings, because comparison between these reactive power components shows the variation of the reverse and the main magnetic fields during unbalanced voltage 
dips and, thus, they inform about the re-magnetization or demagnetization of the wind generators in such transitory phenomena. Instantaneous p-q theories established by Akagi [12] and others [13], widely used for controlling the electronic converters of the wind turbines, was not considered in this study because the well founded objections expressed by Czarnecki in [14,15].

The question is finally where the above defined reactive powers must be registered in order to measure the magnetizing reactive power and, thus, the magnetic field in the doubly fed induction generators. It is known from [16] that when a sudden voltage dip occurs and the currents reach dangerous values, the crowbar system is activated; to avoid the shaft-speed from exceeding the critical value. Doubly fed induction generators operate under such conditions as a squirrel-cage induction generator and, thus, the stator reactive power approximately coincides with the magnetizing reactive power, which defines the magnetic field and, thus, the operation of these machines. This conclusion was applied in Section 4 to analyze operation of one actual wind generator subjected to voltage dips. Practical measures were registered by a Fluke 1760 three-phase power quality recorder and, then, these data were processed by the SIMPELEC software [17] to obtain the reactive powers.

\section{Reactive Power Requirements Established by the Spanish Grid Code}

According to the current and proposed Spanish grid codes [1,2], wind farms and all their components must be operational without any disconnections even with voltage perturbations (in modules and/or angles) present at the net interconnection point, originated by three-phase, two-phase and single-phase to ground faults with the LVRT showed in Figure 1.

Figure 1. Low voltage ride through (LVRT) as specified in Spanish grid code: (a) three-phase faults; (b) single- and two-phase faults.

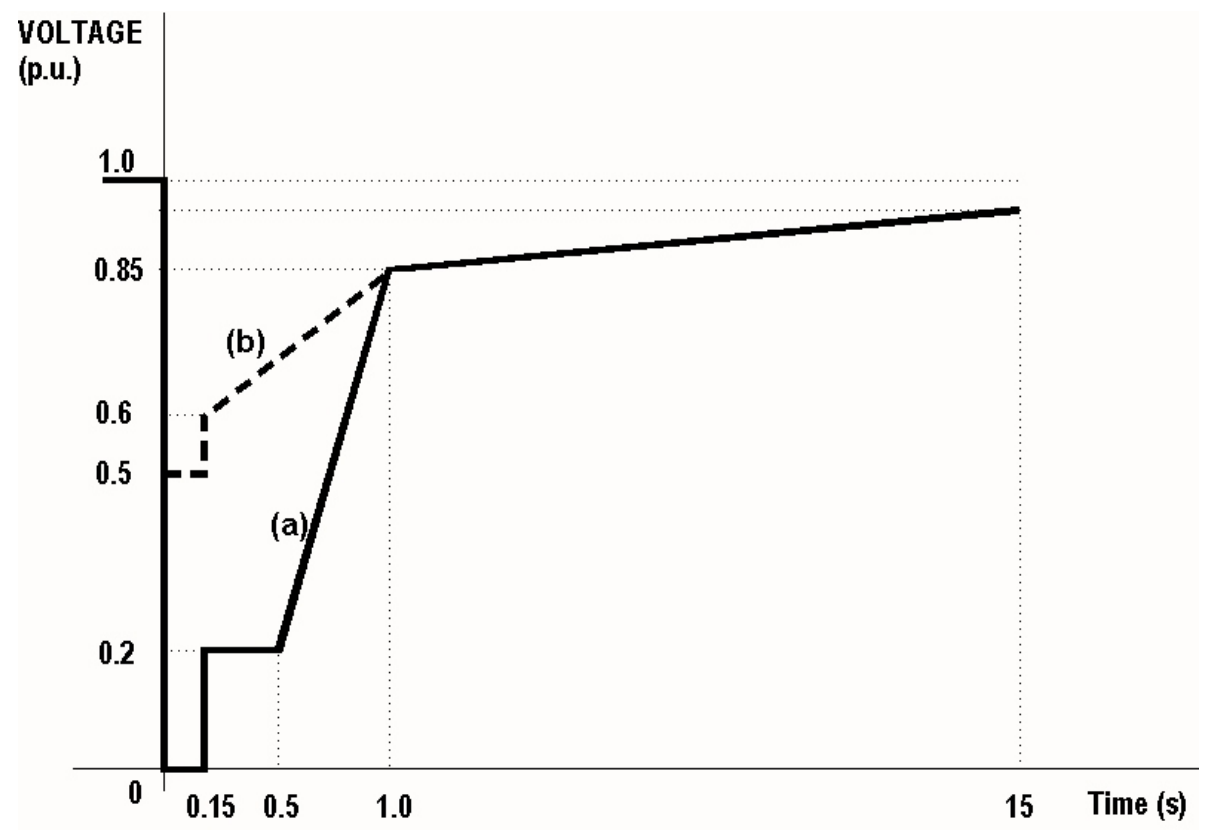


At the same time, the Spanish grid code sets the following limits to the reactive power consumptions:

- Reactive power consumptions are not allowed during the fault and the subsequent recovery period;

- However, for three-phase balanced faults, some reactive power consumptions $40 \mathrm{~ms}$ after the beginning of the fault and $80 \mathrm{~ms}$ after the fault clearance are allowed (Figure 2a);

- For single- and two-phase faults, the Spanish grid code allows some reactive power consumptions during the first $80 \mathrm{~ms}$ after the beginning of the fault and $80 \mathrm{~ms}$ after the fault clearance (Figure 2b).

Reactive power consumptions must not be greater than $60 \%$ of the wind-farm power rated in each network voltage period $(20 \mathrm{~ms})$ for three-phase faults, or greater than $40 \%$ for single- and two-phase faults (Figure 2).

Figure 2. Reactive power limitations established by Spanish grid code: (a) three-phase faults; (b) single- and two-phase faults.

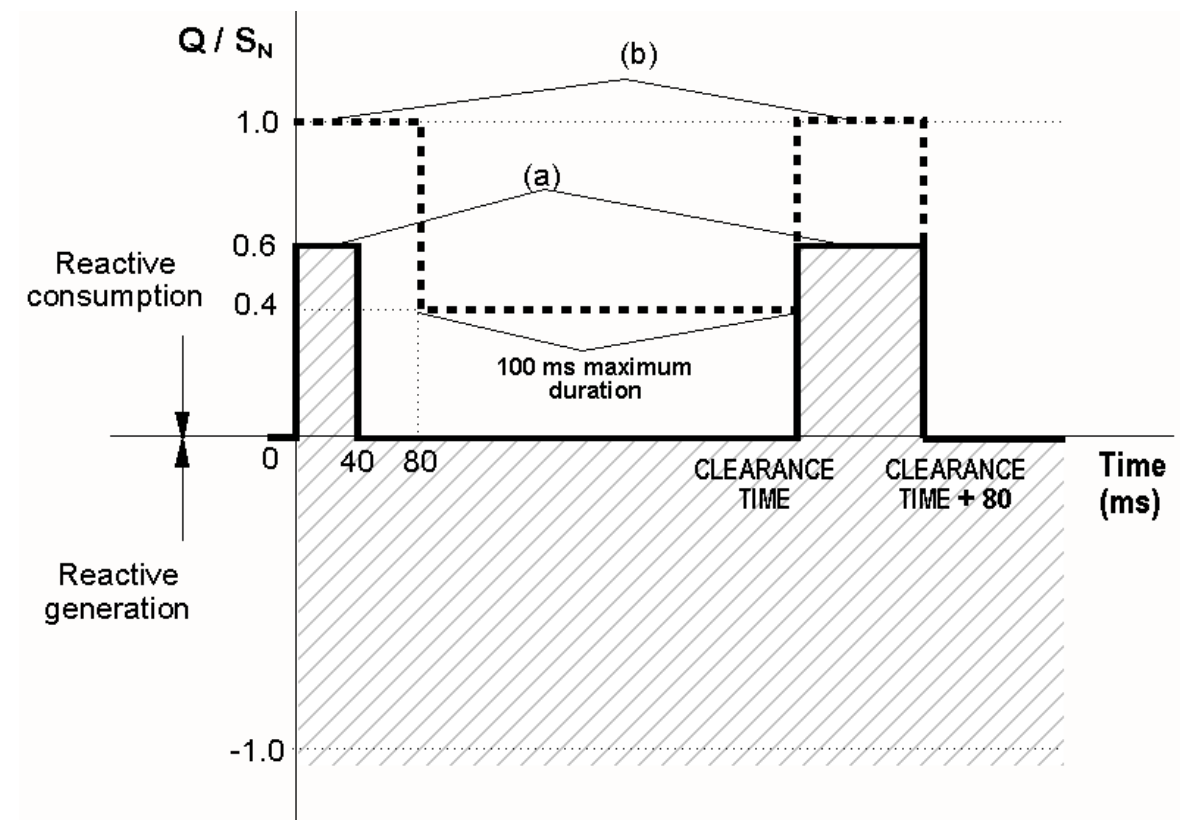

\section{Reactive Power Formulations}

In this section, Delayed Voltage reactive power, Czarnecki's CPC fundamental reactive power and FPRP formulations are defined in one time period of its variability and applied to the equivalent circuit of a wind generator and their load (Figure 3), where no neutral wire is present. These reactive power formulations have been defined in the frequency-domain, but this is not any disadvantage with respect to Akagi's IRP p-q Theory [9], since instantaneous reactive power also must be defined over the entire cycle of its variability in order to identify the power properties of this quantity [12]. 
Figure 3. Wind generator and load at net interconnection point.

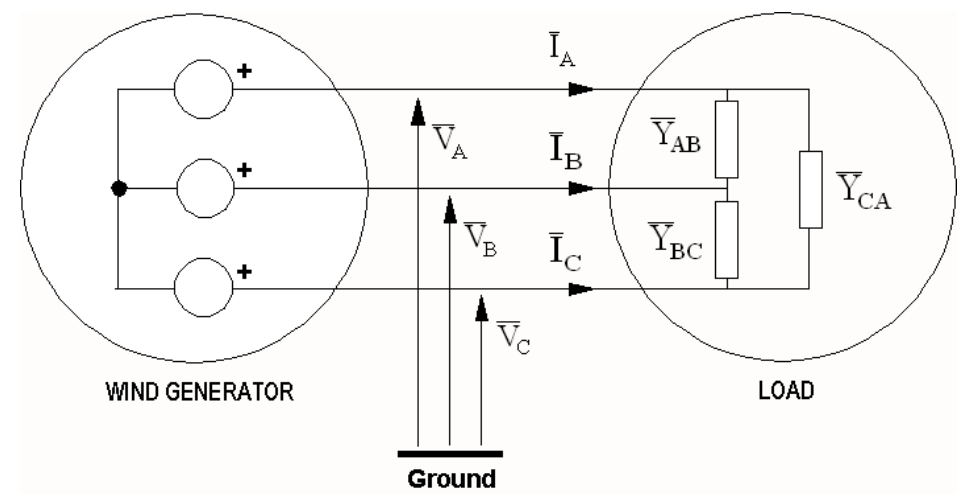

\subsection{Delayed Voltage Reactive Power}

According to this approach, the reactive power is expressed as:

$$
Q=\sum_{z} Q_{z}=\sum_{z}\left(\frac{1}{T} \int_{0}^{T} v_{z}(t-T / 4) \cdot i_{z}(t) \cdot d t\right)
$$

where $T$ is the period of variability of the voltages and currents of each phase $z=\mathrm{A}, \mathrm{B}, \mathrm{C}$.

\subsection{Czarnecki's CPC Fundamental-Frequency Reactive Power}

The traditional reactive power for three-phase three-wire sinusoidal power systems $\left(Q_{1}\right)$ is decomposed by Czarnecki [8] into the reactive power with symmetrically supply voltages $\left(Q_{s}\right)$ and the reactive power due to unbalances $\left(Q_{d}\right)$ as follows:

$$
\begin{aligned}
& Q_{1}=V_{1} \cdot I_{r 1}=-B_{b} \cdot V_{1}^{2}=-\left(B_{e}+B_{d}\right) \cdot V_{1}^{2}=Q_{s}+Q_{d} \\
& Q_{s}=-B_{e} \cdot V_{1}^{2} \\
& Q_{d}=-B_{d} \cdot V_{1}^{2}
\end{aligned}
$$

where:

$$
V_{1}=\sqrt{V_{1 A}^{2}+V_{1 B}^{2}+V_{1 C}^{2}}
$$

Further, the subscript 1 indicates the fundamental-frequency voltages and currents, while $B_{b}, B_{e}$, and $B_{d}$ represent the imaginary parts of the following admittances [6,7], respectively:

$$
\begin{aligned}
& \bar{Y}_{b}=\frac{\bar{S}_{1}^{*}}{V_{1}^{2}}=2 \bar{Y}_{e}-\frac{3}{V_{1}^{2}}\left(\bar{Y}_{B C} V_{1 A}^{2}+\bar{Y}_{C A} V_{1 B}^{2}+\bar{Y}_{A B} V_{1 C}^{2}\right) \\
& \bar{Y}_{e}=\bar{Y}_{A B}+\bar{Y}_{B C}+\bar{Y}_{C A} \\
& \bar{Y}_{d}=\bar{Y}_{e}-\frac{3}{V_{1}^{2}}\left(\bar{Y}_{B C} V_{1 A}^{2}+\bar{Y}_{C A} V_{1 B}^{2}+\bar{Y}_{A B} V_{1 C}^{2}\right)
\end{aligned}
$$

Here, $\bar{S}_{1}^{*}=P_{1}-j Q_{1}$ is the conjugate of the complex power of the three-phase load. 


\subsection{Fundamental-Frequency Positive-Sequence Reactive Power}

As explained in [13-15], the fundamental-frequency positive-sequence phase voltages and currents of the load in the three-phase three-wire power system shown in Figure 3 can be expressed using complex RMS (CRMS) values as follows:

$$
\begin{aligned}
& \bar{V}_{A B+}=V_{A B+} \angle \alpha_{+} \\
& \bar{I}_{A B+}=G_{+} \bar{V}_{A B+}+\bar{B}_{+} \bar{V}_{A B+}+\bar{\delta}_{U} \bar{Y}_{i} \bar{V}_{A B+}
\end{aligned}
$$

The subscripts $(+)$ denote the positive-sequence component and:

$$
G_{+}=\frac{1}{3}\left(\sum_{z=A B, B C, C A} G_{z}\right) \quad B_{+}=\frac{1}{3}\left(\sum_{z=A B, B C, C A} B_{z}\right)
$$

are the load positive conductance $\left(G_{+}\right)$and susceptance $\left(B_{+}\right)$. The phase admittances $\bar{Y}_{z}=G_{z}+\bar{B}_{z}(z=\mathrm{AB}, \mathrm{BC}$, and $\mathrm{CA})$.

Further:

$$
\bar{Y}_{i}=\frac{1}{3}\left(\bar{Y}_{A B}+a^{2} \bar{Y}_{B C}+a \bar{Y}_{C A}\right)=Y_{i} \angle \alpha_{i}
$$

$a=1_{\angle 120^{\circ}}$, is the load admittance for the negative-sequence voltages:

$$
\bar{\delta}_{U}=\frac{\bar{V}_{A B-}}{\bar{V}_{A B+}}=\delta_{U} \angle \alpha_{-}-\alpha_{+}
$$

is the degree of unbalance voltage, and $\alpha_{+}, \alpha_{-}$are the arguments of the fundamental-frequency positive- and negative-sequence line to line voltages.

The fundamental-frequency positive-sequence reactive current of the load $\left(\bar{I}_{A B r+}\right)$ is the component of $\bar{I}_{A B+}$ shifted by $90^{\circ}$ with respect to the voltage $\left(\bar{V}_{1 A B+}\right)$. From (5), it is obtained by the following expression:

$$
\bar{I}_{A B r+}=-j \bar{V}_{A B+} \cdot\left[ \pm B_{+}+\delta_{U} Y_{i} \sin \left(\alpha_{+}-\alpha_{-}+\alpha_{i}\right)=\bar{I}_{r r+}+\bar{I}_{u r+}\right]
$$

The positive sign of $B_{+}$is for inductive loads while the negative sign is for capacitive loads.

The above reactive current can be decomposed into two components. The first component $\left(\bar{I}_{r r_{+}}\right)$is the same reactive current that could be obtained in three-phase power systems supplied by balanced voltages as follows:

$$
\bar{I}_{r r+}=\mp j B_{+} \cdot \bar{V}_{A B+}
$$

This is the reactive current due to the reactive loads $\left(\bar{I}_{r r+}\right)$, and it occurs because of the phase shift between $\bar{V}_{A B+}$ and $\bar{I}_{A B+}$ caused by the reactors of the loads. The second component $\left(\bar{I}_{u r+}\right)$ of the reactive current is expressed as follows:

$$
\bar{I}_{u r+}=-j \bar{V}_{A B+} \cdot\left[\delta_{U} Y_{i} \sin \left(\alpha_{+}-\alpha_{-}+\alpha_{i}\right)\right]
$$

This current occurs because of the phase shift between $\bar{V}_{A B+}$ and $\bar{I}_{A B+}$ caused by the unbalances of both voltages and loads; thus, $\bar{I}_{u r+}$ is the reactive current caused by the unbalances. 
Hence, the fundamental-frequency positive-sequence reactive power absorbed by the load is:

$$
\bar{Q}_{+}=3 \bar{V}_{A B+} \cdot \bar{I}_{A B r+}^{*}=j 3\left[ \pm B_{+}+\delta_{U} Y_{i} \sin \left(\alpha_{+}-\alpha_{-}+\alpha_{i}\right)\right] \cdot V_{A B+}^{2}=\bar{Q}_{r+}+\bar{Q}_{u+}
$$

This power has two components - the traditionally known reactive power due to the reactive loads:

$$
\bar{Q}_{r+}=3 \bar{V}_{A B+} \cdot \bar{I}_{r++}^{*}= \pm j 3 B_{+} V_{A B+}^{2}
$$

and the reactive power due to the unbalances:

$$
\bar{Q}_{u+}=3 \bar{V}_{A B+} \cdot \bar{I}_{u r+}^{*}=j 3\left[\delta_{U} Y_{i} \sin \left(\alpha_{+}-\alpha_{-}+\alpha_{i}\right)\right] \cdot V_{A B+}^{2}
$$

It can be easily demonstrated that the reactive powers expressed above are the same reactive powers supplied by the wind generator. First, the reactive power is a conservative quantity. Second, mathematically, from Stokvis-Fortescue transformation, it is known that the fundamental-frequency positive-sequence phase to ground voltages (Figure 3) can be expressed as a function of the load phase voltages as follows:

$$
\bar{V}_{A+}=\frac{\bar{V}_{A B+}}{\sqrt{3}} \angle-30^{\circ}
$$

Further, similarly, the fundamental-frequency positive-sequence reactive line-currents supplied by the generator are:

$$
\bar{I}_{A r+}=\sqrt{3} \cdot \bar{I}_{A B r+} \angle-30^{\circ}
$$

\subsection{Reactive Power Components}

The reactive powers formulated above were defined by considering the reactive phenomena under different voltage and current conditions. Delayed Voltage reactive power $(Q)$ was established for voltages and currents at all frequencies; Czarnecki's CPC reactive power was defined for the fundamental-frequency voltages and currents; and FPRP was defined for the fundamental-frequency positive-sequence voltages and currents. Thus, the differences between these three reactive powers provide information about the effects of different phenomena, which can be useful to evaluate the operation of wind generators.

The following reactive powers and their utilities may then be established:

(1) Non-fundamental frequency reactive power $\left(Q_{H}\right)$ is the difference between Budeanu's and Czarnecki's reactive powers as follows:

$$
Q_{H}=Q-Q_{1}
$$

This quantity determines the distorted voltage and current effects.

(2) Negative-sequence reactive power $\left(Q_{-}\right)$is the difference between Czarnecki's and Emanuel reactive powers:

$$
Q_{-}=Q_{1}-Q_{+}
$$


This quantity defines the power effects caused by the negative-sequence voltages and currents, and it comprises two components:

(3) Negative-sequence reactive power due to the load $\left(Q_{r-}\right)$ :

$$
Q_{r-}=Q_{s}-Q_{r+}
$$

This quantity measures effects of the reactive loads caused by the negative-sequence voltages.

(4) Negative-sequence reactive power caused by the unbalances $\left(Q_{u^{-}}\right)$:

$$
Q_{u-}=Q_{d}-Q_{u+}
$$

The above quantity shows the reactive power effects caused by increases in the negative-sequence currents due to the unbalances of both supply voltages and reactive loads.

These reactive powers are also used to define magnetic fields in the wind generators, which are related with each their operation involved phenomena.

\section{Practical Experiences}

A remarkable two-phase voltage dip was considered for analyzing the operations of one actual wind generator. The study is made through the observation of the magnetizing reactive power, since this quantity determinates the magnetic field of the wind generators. In the normal operation, the magnetizing reactive power of the doubly fed wind generators is related with the reactive power measured at the stator and varies inversely with the slip(s) of these machines. But, in presence of voltage dips, the crowbar is applied to the rotor windings (Figure 4) and, thus, wind generators operate as the squirrel-cage induction generators [16], where stator reactive power approximately is equal to the magnetizing reactive power. Hence, for the experimental analysis showed in this section, the reactive power formulations expressed in the previous section are applied to the data measured at the stator.

Figure 4. Doubly fed induction generator and crowbar system.

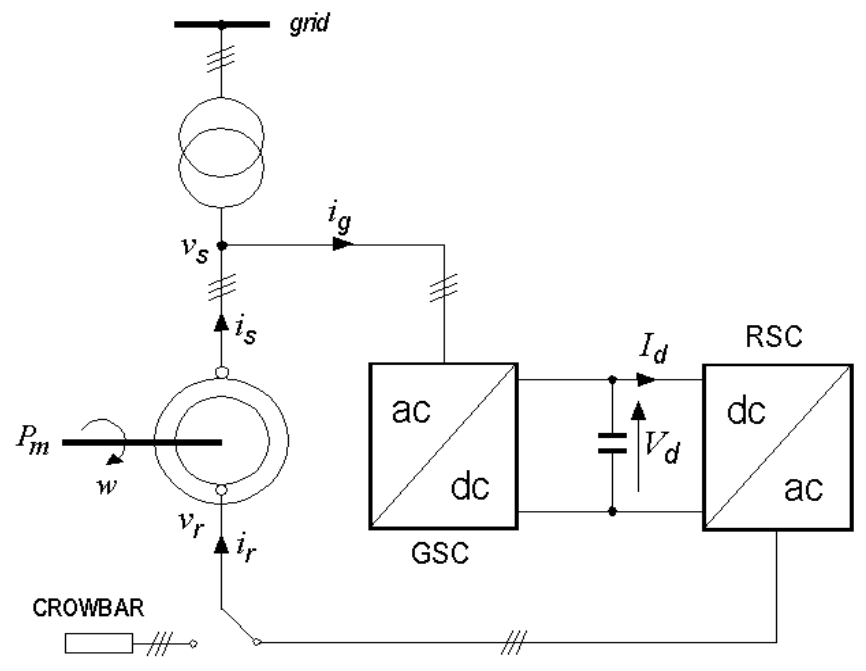


Stator of the double-fed induction generator is directly connected to the grid through a power transformer (Figure 4), and they have the following electrical rated characteristics: power $=660 \mathrm{~kW}$ and line-to-line voltages $=690 \mathrm{~V}$. The data were recorded using the power quality recorder Fluke 1760 (Figure 5) and subsequently processed by the SIMPELEC software [17].

Figure 5. Connection of the Fluke 1760 three-phase power quality recorder.

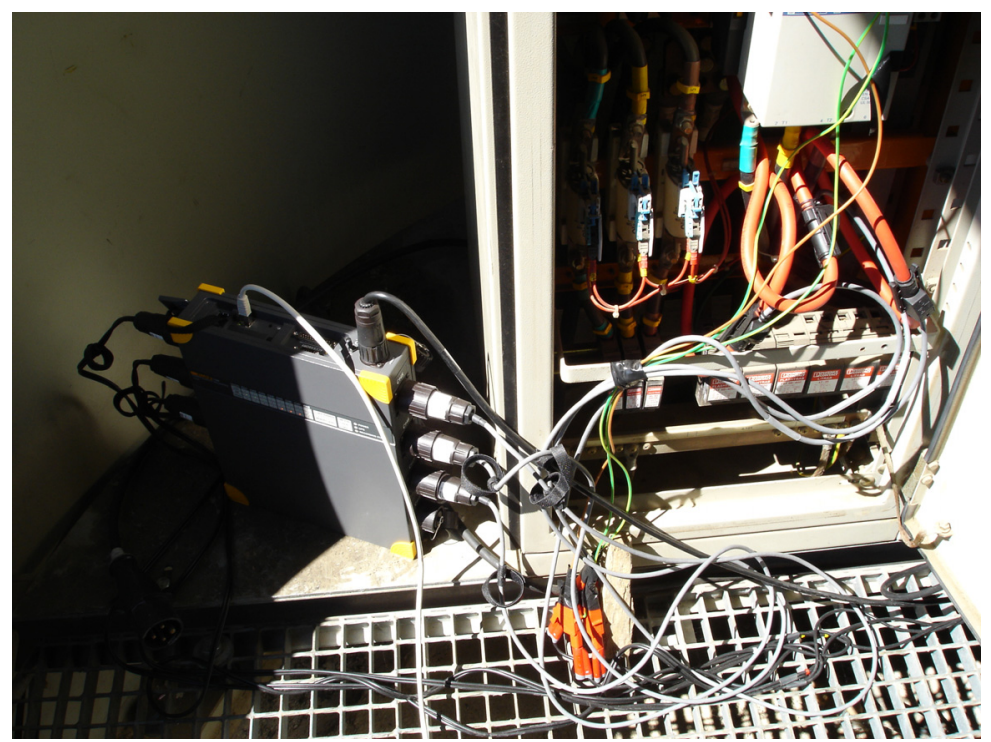

Figure 6 shows an 80-ms two-phase registered voltage dip. The voltages of the A and B-phases decreased to less than 40 and $30 \%$, respectively, of their rated values, while the C-phase voltage did not decrease to less than $10 \%$; hence, the failure affected only two phases (according to the voltage dip definition included in the Std. UNE-IEC/TR 61000-2-8 IN [3]).

Figure 6. Two-phase voltage dip, RMS voltages (p.u.).

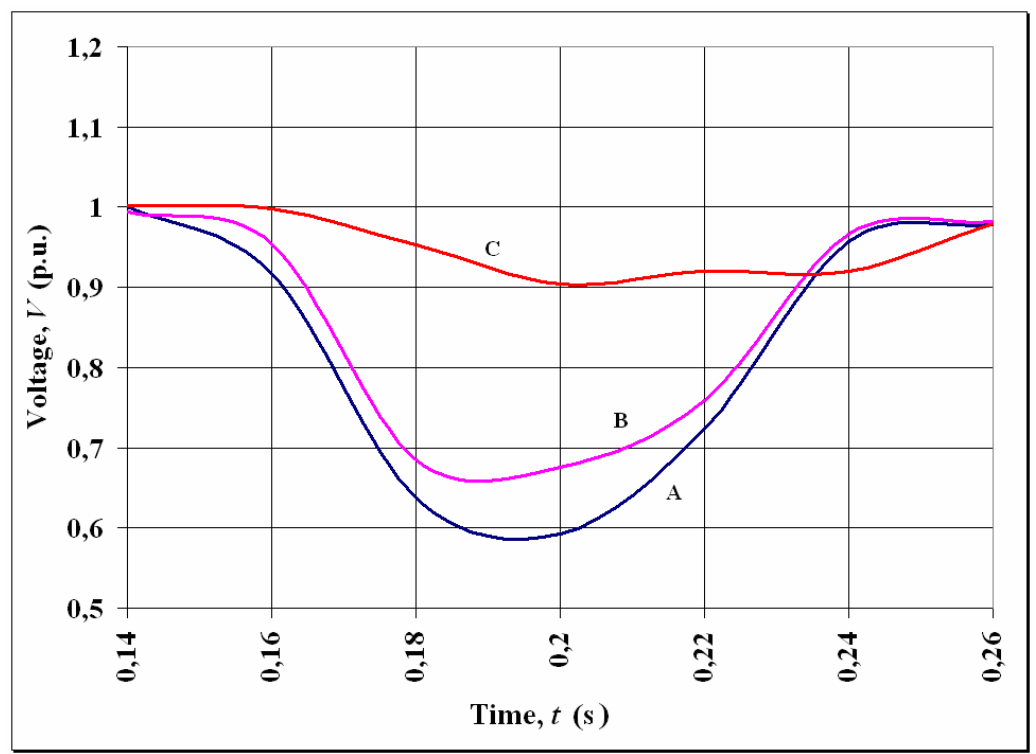


The reactive powers obtained by DV method, Czarnecki's CPC theory, and FPRP formulations are shown in Figure 7. These reactive powers were supplied by the wind generator (positive sign, according to definition included in [3]). DV and Czarnecki's reactive powers had similar values before and after the perturbation (165 kVAr before the beginning of the voltage dip and $120 \mathrm{kVAr}$ after the clearance of the voltage dip); however, during the perturbation, the three analyzed reactive powers had different values. Czarnecki's reactive power had maximum values of $275 \mathrm{kVAr}$, while FPRP reduced (Figure 7); but, since no FPRP consumptions occurred during the voltage dip period, the reactive power requirements specified by the Spanish grid code were verified.

Notice that only the values of FPRP are applied to verify the accomplishment of the Spanish grid code requirements; however, other reactive powers could be used to analyze operation of the wind generators.

Figure 7. Reactive powers: (a) Delayed Voltage, (b) Czarnecki, (c) FPRP.

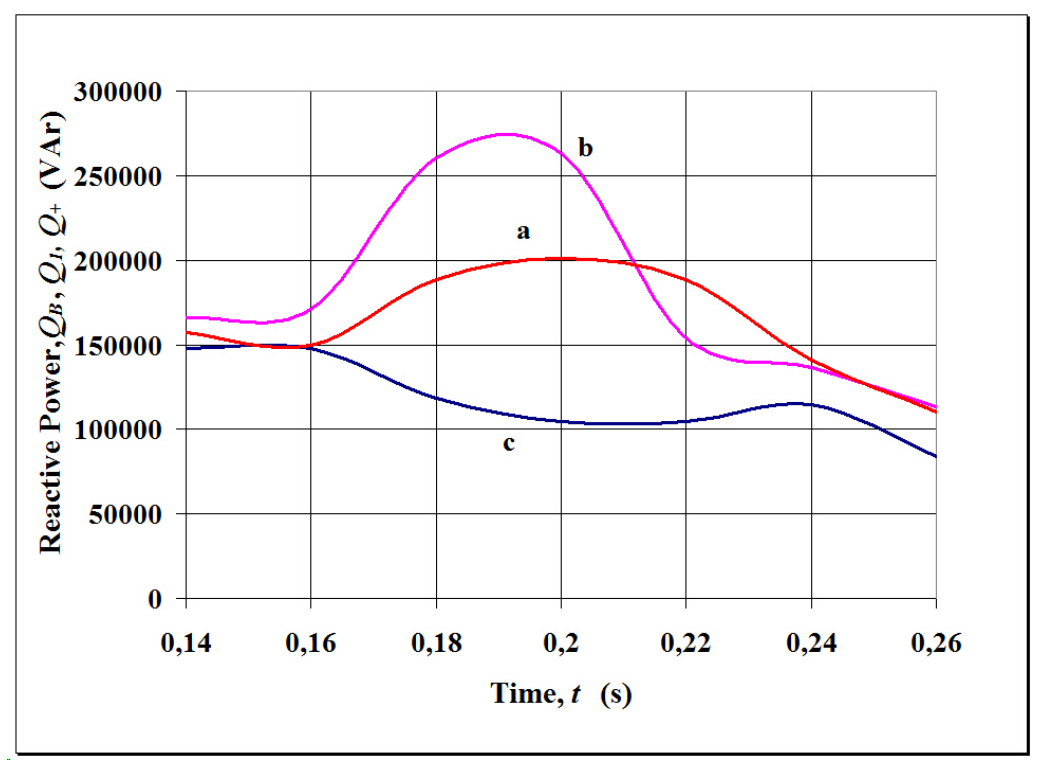

The Delayed Voltage (DV) reactive power $(Q)$ is obtained through all voltage and current components, while Czarnecki's CPC fundamental reactive power $\left(Q_{1}\right)$ is expressed by the voltages and currents of fundamental-frequency. Measured values of $Q$ were different that those of Czarnecki's $\left(Q_{1}\right)$ during the perturbation (Figure 7). These reactive powers, $Q$ and $Q_{1}$, define different magnetic fields, which rotate at different speeds and cause some distinctive effects. The difference between $Q$ and $Q_{1}$ has been called in this paper as the non-fundamental reactive power $\left(Q_{H}\right)$, which defines the harmonic magnetic fields in the wind generator, originates harmonic electromagnetic forces and torques and generally decreases the generator efficiency. Non-fundamental reactive power was absorbed by the wind generator in the first half of the voltage dip, and the maximum value was $75 \mathrm{kVAr}$ (Figure 8). During the second half of the voltage dip, non-fundamental reactive power was supplied by the wind generator; the maximum value was $35 \mathrm{kVAr}$. The sign, positive or negative, of the values of $Q_{H}$ do not provide fundamental information about the magnetic field defined by this reactive power. The magnetic fields corresponding to each individual harmonic have significance; the speed and direction of rotation for each harmonic magnetic field is determined by its own frequency and its sign (positive or negative) indicates the direction of the electromagnetic forces and torques. Existence of 
the non-fundamental reactive power informs about the deformation of the magnetic field and its value only should be considered as a reference about the distortion importance. In the present experience, non-fundamental reactive power reached comparable values with the fundamental positive-sequence reactive power $\left(Q_{+}\right)$; this implied that harmonic magnetic fields strongly perturbed the operation of wind generators during the unbalanced voltage dip.

Figure 8. Non-fundamental reactive power.

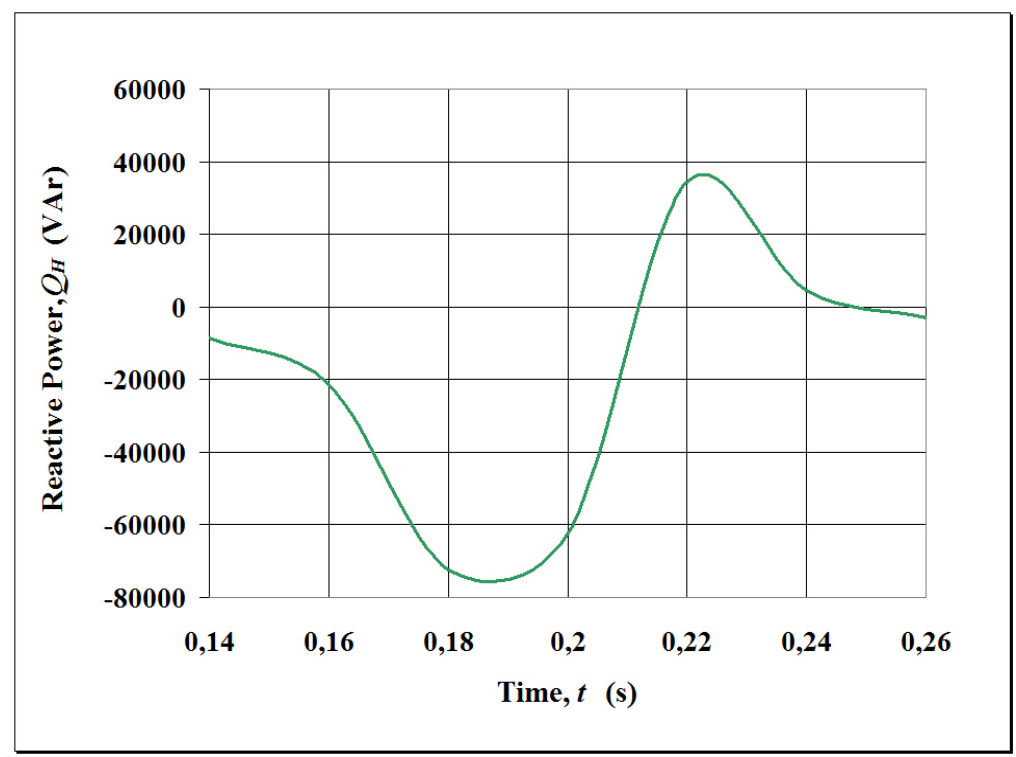

Czarnecki's CPC fundamental reactive power $\left(Q_{1}\right)$ and FPRP $\left(Q_{+}\right)$had great differences during the voltage dip period (Figures 7, 9 and 11). This is because the effect of the voltage unbalances, which determinate the negative reactive power $\left(Q_{-}\right)$, whose evolution is showed in Figure 10a.

Figure 9. Czarnecki's CPC fundamental reactive powers: (a) total, (b) due to reactive loads, (c) caused by imbalances.

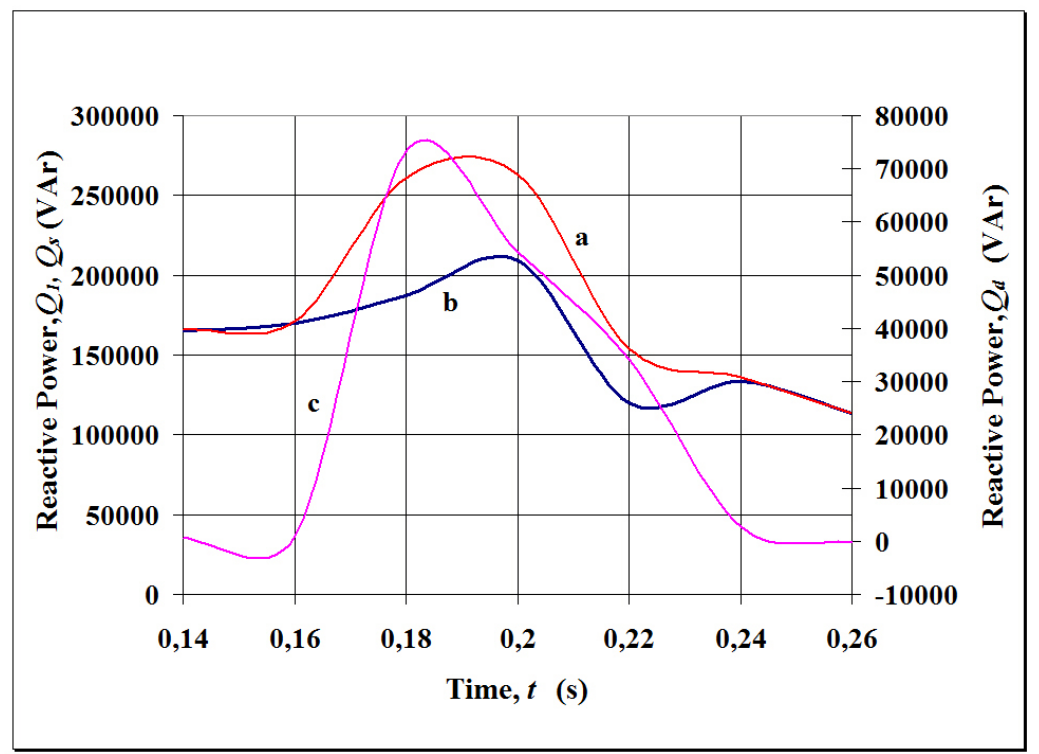


This reactive power component defines the reverse magnetic field, which rotates with the same speed and opposite direction that the main magnetic field of the wind generator and creates reverse

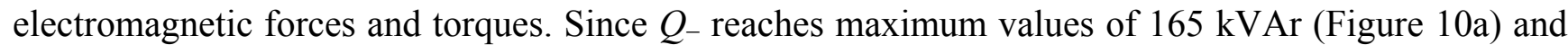
FPRP $\left(Q_{+}\right)$is $100 \mathrm{kVAr}$, the wind generator was subjected in this experience to large electrodynamic forces, due to the voltage imbalances, during the voltage dip. However, in spite of $Q_{-}>Q_{+}$, reverse torques were less than direct torques, since values of these electromagnetic torques are inversely proportional to 2-s and s, respectively, where s is the slip of the wind generator.

In the other hand, decomposition of $Q_{-}$and $Q_{+}$into two components (Figure 10 and Figure 11, respectively) provides additional information, which can help us to know the causes of these large reverse forces and magnetic fields. In the present experience, the negative reactive power component due to the reactive loads $\left(Q_{r-}\right)$ and the negative reactive power component caused by the voltage unbalances $\left(Q_{u^{-}}\right)$have comparable values during the voltage dip (Figure 10), but $Q_{u^{-}}$is greater than $Q_{r}$ - at the beginning and in the end of the voltage dip. This one implies the reverse torques caused by the voltage unbalances have more importance during these periods than the reverse torques due to the reactive loads, and the contrary occurs in the middle of the voltage dip where $Q_{r^{-}}>Q_{u^{-}}$, in this experience (Figure 10). Anyway, negative reactive power components are responsible of poor wind generator operations.

Figure 10. Negative-sequence reactive powers: (a) total, (b) due to reactive loads, (c) caused by imbalances.

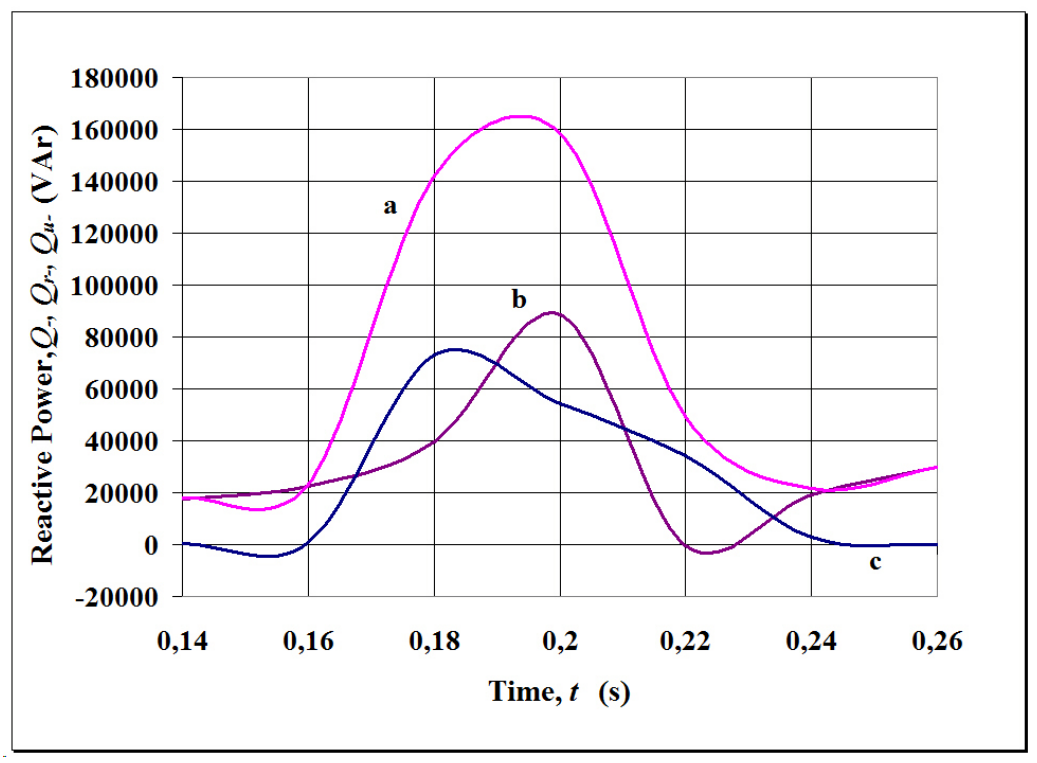

Figure 11 shows that the FPRP $\left(Q_{+}\right)$decreased during the voltage dip as a result of the existence of a reactive power component caused by the unbalances $\left(Q_{u+}\right)$, which is absorbed by the generator (Figure 11c) and partially compensates the FPRP due to the reactive loads (with balanced voltages, $\left.Q_{r^{+}}\right)$. This reduction reached a third part of the fundamental positive-sequence reactive power $\left(Q_{+}\right)$, thereby causing a sudden demagnetization of the generator when the main magnetic field (created by $Q_{+}$) decreased. As a result, the relative importance of the negative-sequence magnetic fields that were established by $Q_{-}$(Figure 10) increased. 
Figure 11. Positive-sequence reactive powers: (a) total, (b) due to reactive loads, (c) caused by unbalances.

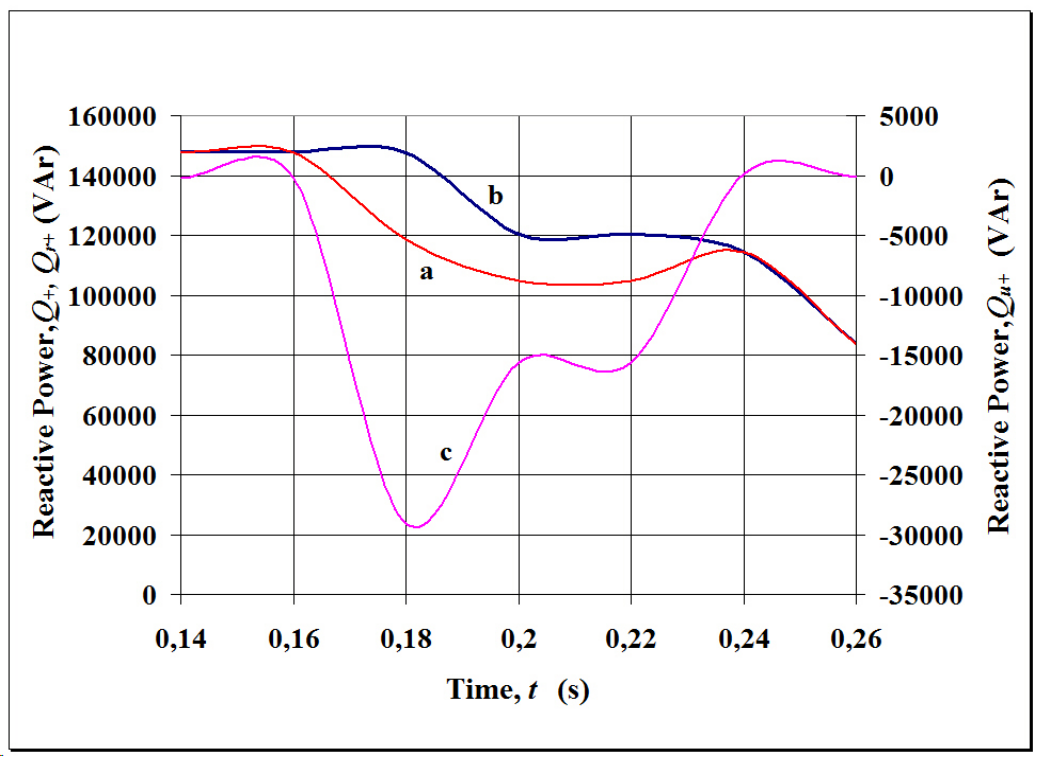

In conclusion, the operation of the wind generator was considerably perturbed during the two-phase unbalanced voltage dip and this perturbation can be explained by using the magnetic fields defined by the magnetizing reactive powers. The primary reasons for the poor generator operations are as follows: (a) unbalanced voltages and loads that decreased the magnetic field of the main generator (created by $Q_{+}$) and increased the reverse magnetic field (due to $Q_{-}$), and (b) the distorted voltages and loads that caused harmonic magnetic fields with different speeds (some with different rotation senses) than the main magnetic field. However, wind generator could support the voltage dip without disconnection.

\section{Conclusions}

Fundamental-frequency positive-sequence reactive power (FPRP) included in the IEEE Standard 1459 and recently proposed by the IEC 61400-21:2008 and other wind farms standards should be supplemented with other reactive power formulations in order to obtain a complete and detailed analysis of the wind-generator operations in unbalanced and/or non-sinusoidal voltage conditions. This is because the main magnetic field of the wind generators, defined by the FPRP, is perturbed by the magnetic fields originated by voltage imbalances and distortions, when subjected to voltage dips.

For this purpose, the use of the non-fundamental and the negative-sequence reactive powers, established in this paper by comparing Delayed Voltage reactive power, Carnecki's CPC fundamental-frequency reactive power and FPRP, and the decomposition of Czarnecki's and FPRP into two components, due to the reactive loads and caused by the imbalances, provide additional information about wind generator operation in presence of voltage perturbations. The non-fundamental reactive power characterizes the presence of harmonic magnetic fields, while negative-sequence reactive power defines fundamental reverse magnetic fields. These magnetic fields perturb the main magnetic field and thereby explain the poor operations of wind generators and failures caused in the presence of unbalanced and distorted voltage dips. Also, it was observed on the application example 
that FPRP components, due to the reactive loads and caused by the imbalances, could have the same or opposite sign and, thus, increases or decreases of the main magnetic fields, responsible of the re-magnetization or demagnetization, respectively, of the wind-generators, could be otherwise explained.

\section{References}

1. Spanish Grid Code, 2006. Response Requirements in Front of Voltage Dip at Wind Farms Utilities; BOE No. 254; Industry, Tourism and Commerce Spanish Ministry: Madrid, Spain, 2006; pp. 37017-37019.

2. Offprint form P.O.12.2 outline, 2008. Technical Requirements for Wind Power and Photovoltaic Installations and Any Generating Facilities Whose Technology Does Not Consist on a Synchronous Generator Directly Connected to the Grid. Utilities Connected to the Transport Grid and Generating Equipment: Minimum Design Requirements, Equipment, Operation, Deployment and Security; Working document for wind and solar companies, Spanish Ministry of Industry and Energy: Madrid, Spain, 2008.

3. IEC 61400-21:2008, UNE-EN 61400-21:2009. Wind turbines-Part 21: Measurement and Assessment of Power Quality Characteristics of Grid Connected Wind Turbines; AENOR: Madrid, Spain, 2009.

4. León-Martínez, V. Integral Method for the Efficiency and Power Quality Improvement on the Electric Energy Supply at Low Voltage. Ph.D. Thesis, Universidad Politécnica de Valencia. Valencia, Spain, September, 1997.

5. León-Martínez, V.; Giner-García, J.; Montañana-Romeu, J.; Cazorla-Navarro, A. Efficiency in electrical installations. New power definitions. Mundo Electrónico 2001, 322, 28-32.

6. Emanuel, A.E. Apparent powers definitions for three-phase systems. IEEE Trans. Power Deliv. 1999, 14, 767-772.

7. Institute of Electrical and Electronics Engineers. IEEE Trial Use Standard Definitions for the Measurement of Electric Power Quantities under Sinusoidal, Non-Sinusoidal, Balanced, or Unbalanced Conditions; IEEE Standard 1459-2010; New York, NY, USA, 2010.

8. Czarnecki, L.S. Powers of asymmetrically supplied loads in terms of the CPC power theory. Electr. Power Qual. Utiliz. J. 2007, 13, 97-104.

9. León-Martínez, V.; Montañana-Romeu, J.; Giner-García, J.; Cazorla-Navarro, A.; Roger-Folch, J.; Graña-López, M.A. Power Quality Effects on the Measurement of Reactive Power in Three-Phase Power Systems in the Light of the IEEE Standard 1459-2000. In Proceedings of the 9th International Conference Electric Power Quality and Utilization, Barcelona, Spain, 7-9 October, 2007.

10. León-Martínez, V.; Montañana-Romeu, J. Method and System for Calculating the Reactive Power in Disturbed Three-Phase Networks. WIPO Patent Application WO/2010/007186, July, 2009.

11. Leon-Martinez, V.; Montañana-Romeu, J. Active and Reactive Power Formulations for Grid Code Requirements Verification. In From Turbine to Wind Farms-Technical Requirements and Spin-Off Products; Krause G., Ed.; InTech: 2011, pp. 41-62. Available online: http://www.intechopen.com/articles/show/title/active-and-reactive-power-formulations-for-gridcode-requirements-verification. 
12. Akagi, H.; Kanazawa, Y.; Nabae, A. Instantaneous reactive power compensators comprising switching devices without energy storage components. IEEE Trans. Ind. Appl. 1984, 20, 625-630.

13. Kim, H.; Blaabjerg, F.; Bak-Jensen, B. Spectral analysis of instantaneous powers in single-phase and three-phase systems with use of p-q-r theory. IEEE Trans. Power Electron. 2002, 17, 711-720.

14. Czarnecki, L.S. Comparison of instantaneous reactive power p-q theory with theory of the current's physical components. Electr. Eng. 2003, 85, 21-28.

15. Czarnecki, L.S. On some misinterpretations of the instantaneous reactive power p-q theory. IEEE Trans. Power Electron. 2004, 19, 828-836.

16. Salles, M.B.C.; Hameyer, K.; Cardoso, J.R.; Grilo, A.P.; Rahmann, C. Crowbar system in doubly fed induction wind generators. Energies 2010, 3, 738-753.

17. Montañana-Romeu, J.; León-Martínez, V. SIMPELEC 5: Software for the measurement of powers, energies, efficiency and power quality in electrical installations. Protected by the Universidad Politécnica de Valencia, Valencia, Spain, February 2008.

(C) 2011 by the authors; licensee MDPI, Basel, Switzerland. This article is an open access article distributed under the terms and conditions of the Creative Commons Attribution license (http://creativecommons.org/licenses/by/3.0/). 